\title{
Similarity Measures for Binary and Gray Level Markov Random Field Textures
}

\author{
Abdurrahman Carkacıoglu, and Fatoş T. Yarman-Vural \\ Department of Computer Engineering \\ Middle East Technical University \\ E-mail: vural@ceng.metu.edu.tr
}

\begin{abstract}
In this study a new set of texture measures, namely, Clique Length and its moments are introduced. These measures are defined employing new concepts which agrees with the human visual system. The simulation experiments are performed on binary and gray level MRF texture alphabet to quantify the data by the $\mathrm{k}^{\text {th }}$ moment of Clique Length. Experimental results indicate that the introduced measures identify the visually similar textures much better than the mathematical distance measures.
\end{abstract}

\section{INTRODUCTION}

Loosely speaking, texture can be defined as a stochastic, possibly periodic, two dimensional image field. In recent computer vision literature there has been an increasing interest in the use of statistical techniques for modeling and processing the textured image. Some of this work has been directed towards the application of Markov Random Fields (MRF) in texture modeling, classification and restoration of noisy and textured images [1],[2].

Texture generation using the MRF model is a classical problem. Using different set of parameters, it is possible to generate extremely wide class of textures. However, it is not easy to generate a desired form of texture, since the relationship between the model parameters and certain features of texture is not a linear one.

In this study, a set of measures is introduced to quantify the similarity of textures which can be modeled by Markov Random Fields (MRF). First, a brief explanation of MRF model is given in Section 2. Texture realization problem is addressed in Section 3 . Then, based on the theoretical studies and various observations, a set of texture measures are defined in Section 4. In Section 5, simulation experiments are performed to determine the relation between the data type, model parameters and the texture measures defined in Section 4. Section 6, concludes the paper by discussing the proposed texture measures and commenting on the directions of the feature research. 


\section{MARKOV RANDOM FIELDS (MRF) FOR TEXTURE MODELING}

Consider the random field defined over a finite lattice of points $(i, j), L=$ $\left\{(i, j): 1<i<N_{1}, 1<j<N_{2}\right\}$. A collection of subsets of $L$ described as, $\eta=\left\{\eta_{i j}:(i, j) \in L, L\right.$ $\left.\supset \eta_{i j}\right\}$, is a neighborhood system on $L$ iff $\eta_{i j}$, the neighborhood of pixel $(i, j)$, is such that (a) $(i, j) \notin \eta_{i j},(b)(k, l) \in \eta_{i j}$, for any $(i, j) \in \eta_{k l}$.

A random field $X=\left\{X_{i j}\right\}$, defined over the lattice $L$, is a Markov Random Field (MRF) with respect to the neighborhood system $\eta$, iff the distribution of $X, P(X=x) \geq$ $0, \forall x$ and $P\left(X_{i j}=x_{i j} \mid\left\{X_{k l}=x_{k d},(k, l) \in L,(k, l) \neq(i, j)\right\}\right)=$

$$
P\left(X_{i j}=x_{i j} \mid\left\{X_{k l}=x_{k d},(k, l) \in \eta_{i j}\right\}\right) \text {, for all }(i, j) \in L \text {. }
$$

The above theoretical background is used to generate textures and estimate the model parameters. The reader is referred to [1], [2], [3] for a more detailed description of Markov Random Field texture realization problem.

\section{TEXTURE SIMILARITY MEASURES}

Because of its complexity and tremendous amount of variations, there is no clear-cut mathematical definition of texture. Therefore, a measure of texture which gives an idea about the data type is highly desirable. This measure would be very useful to give a quantitative idea about the texture similarity among the textures, in texture classification problem. In the following, behind a series of definitions, a new set of texture measures is introduced. First, a simple tool for measuring various properties of texture, called base clique is defined. For this purpose, the general concept of clique [4], [5] and neighborhood is utilized.

Definition 1: Base Clique: Given a seed pixel ( $i, j$ ) in a neighborhood system $\eta$, the base clique of $(\mathrm{L}, \eta)$ denoted by a pair of pixels $\mathbf{B}_{\mathrm{p}}(\mathbf{i j}, \mathbf{k l})$ where $\mathrm{p}=1 . . \mathrm{P}$, is a subset of the Lattice $L$ such that (a) $(i, j) \neq(k, l), i j \in \eta_{k l}$ and (b) Pixels $(i, j)$ and $(k, 1)$ satisfies $\left|x_{i j}-x_{k l}\right|<\varepsilon$, for a given $\varepsilon>0$, where $P$ indicates the number of distinct base cliques ( see:Fig. 1)

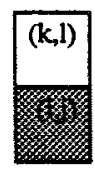

1

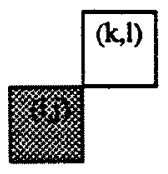

2

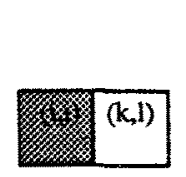

3

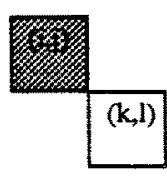

4

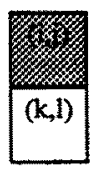

5

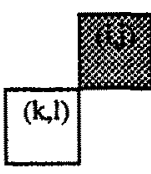

6

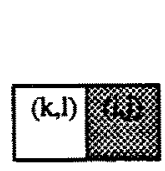

7

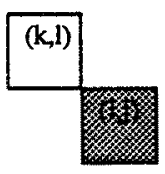

8

Figure 1. $\eta^{2}$ neighborhood systems and its base cliques $B_{P}, P=1,2.8$.

Definition 2: Base Clique Chain: Given pixel $(\mathrm{i}, \mathrm{j})$ as a seed, the base clique chain $\mathbf{C}_{\mathbf{P}}(\mathrm{ij})$ is the connected chain of pixels with the same base clique ,

$$
\forall \mathrm{p}, \quad \mathrm{C}_{\mathrm{p}}(\mathrm{ij})=\left\{\mathrm{B}_{\mathrm{p}}(\mathrm{ij}, \mathrm{kl}) \cup \mathrm{B}_{\mathrm{p}}(\mathrm{kl}, \mathrm{mn}) \cup \mathrm{B}_{\mathrm{p}}(\mathrm{mn}, . .) \cup . . \mathrm{B}_{\mathrm{p}}(\mathrm{qr}, \mathrm{st})\right\}
$$


where (qr,st) is the last connected pair of pixels, with the base clique $B_{p}$. Briefly base clique chains are the line likes starting from the seed pixel and ending at the last connected pair of pixel.

Definition 3 : Given $(\mathrm{i}, \mathrm{j})$ as a seed, $n^{\text {th }}$ Order Clique Chain is

$$
O_{Z}^{n}(i j)=\underset{k \in z}{\cup} C_{k}(i j)
$$

where $\mathrm{z}$ is the $\mathrm{n}$-combination of the integers $1, \ldots, P$. Note that the number of $\mathrm{n}^{\text {th }}$ order clique chain is $P ! /[(P-n) !$, $n !]$ where $P$ indicates the number of distinct base cliques.

$n^{\text {th }}$ Order Clique Chain measures give us cornering information in a given texture.

For example, a second order clique chain is a combination of two base clique chains and defined as $\mathrm{O}_{\mathrm{pq}}^{2}(\mathrm{ij})=\mathrm{C}_{\mathrm{p}}(\mathrm{ij}) \cup \mathrm{C}_{\mathrm{q}}(\mathrm{ij})$.

Note that there are 28 distinct second order clique chains for a seed pixel $(\mathrm{i}, \mathrm{j})$. Figure 2 illustrates some of the second and third order clique chains.

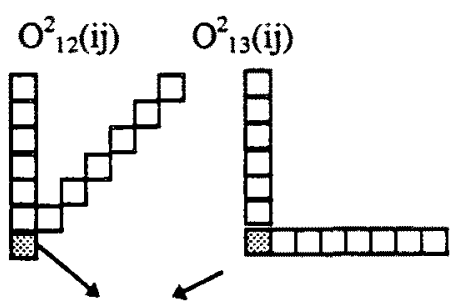

Pixel $(\mathrm{i}, \mathrm{j})$ as a seed

(a)

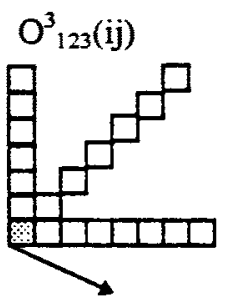

Pixel $(\mathrm{i}, \mathrm{j})$ as a seed

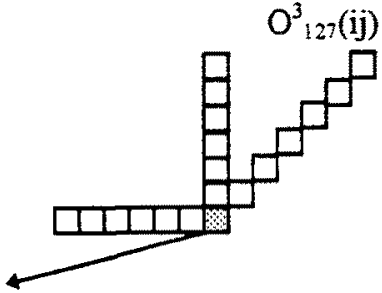

(b)

Figure 2: Some of the a) second and b) third order clique chains, respectively.

Definition 4: Given pixel $(i, j)$ as a seed, and its base clique chain $C_{p}(i j)$, the base clique length $L_{p}(i j)$ is the number of the pixels in $C_{p}(i j)$.

In other words, the base clique length, $\mathbf{L}_{\mathbf{p}}(\mathrm{ij})$, is the number of connected chain of pixels formed by the elements of the base clique chain, $\mathrm{C}_{\mathrm{p}}(\mathrm{ij})$.

In a textured image, the base clique length, $\mathbf{L}_{\mathbf{p}}(\mathrm{ij})$ of each base clique chain is computed by counting the pair of pixels as long as they belong to the same base clique chain set, $\mathrm{C}_{\mathrm{p}}(\mathrm{ij})$.

Definition 5: Given a seed pixel $(i, j)$ and its $n^{\text {th }}$ order clique chain $\mathrm{O}_{z}{ }_{z}(\mathrm{ij})$, the $\mathbf{n}^{\text {th }}$ order clique length $L^{n}{ }_{z}(i j)$, for $n>1$, is defined as $L_{z}{ }^{n}(i j)=\min \left\{L_{p}(i j)\right\}$ where $p \in Z$. 
The effect of $\mathrm{n}^{\text {th }}$ order clique on the human visual system can be measured by considering the minimum length of the $n^{\text {th }}$ order clique chain components, $L_{p}(i j)$. Suppose that, we have $O^{2}{ }_{12}(\mathrm{ij})$ with base clique lengths $\mathrm{L}_{1}(\mathrm{ij})=1, \mathrm{~L}_{2}(\mathrm{ij})=8$ and $\mathrm{O}^{\prime 2}{ }_{12}$ (ij) with base clique lengths $L_{1}^{\prime}(\mathrm{ij})=4$ and $\mathrm{L}_{2}^{\prime}(\mathrm{ij})=4$. For our visual system, $\mathrm{O}^{2}{ }_{12}(\mathrm{ij})$ caries more second order information than $\mathrm{O}_{12}^{2}(\mathrm{ij})$.

Due to the stochastic nature of the texture, $\mathrm{L}_{\mathrm{z}}{ }^{\mathrm{n}}(\mathrm{ij})$ can be considered as a random variable. Therefore, moments, especially, second order statistics of $L_{z}{ }^{n}(i j)$ can give us an idea about the appearance of the texture.

Definition 6: $\mu^{\mathrm{n}}{ }_{\mathrm{z}}$ is the second order moment of $\mathrm{L}_{\mathrm{z}}^{\mathrm{a}}(\mathrm{ij})$ (Clique Length)

$$
\mu^{\mathrm{n}}{ }_{\mathrm{Z}}=\mathrm{E}\left[\left[\mathrm{L}_{\mathrm{Z}}^{\mathrm{n}}(\mathrm{ij})\right]^{2}\right] \approx(1 / N) \Sigma\left[\mathrm{L}_{\mathrm{z}}^{\mathrm{n}}(\mathrm{ij})\right]^{2}
$$

where $\mathrm{E}$ indicates the expected value operator and $\mathrm{N}$ indicates the total number of clique chain sets in Clique type $\mathrm{O}_{2}^{*}$ (ij) . Note that the number of the $\mu^{*}$ is the same as the number of the $\mathrm{n}^{\text {th }}$ order clique chains in a given texture.

Notice that each clique type is represented by a single second order moment. $\mu^{k, n}{ }_{2}$ is the $k^{\text {th }}$ order moment of $\mathrm{L}_{\mathrm{Z}}^{\mathrm{z}} \mathrm{(ij)}$ (Clique Length)

$$
\mu_{z}^{k+m}=E\left[\left[L_{z}^{n}(i j)\right]^{k}\right] \approx(1 / N) \Sigma\left[L_{z}^{n}(i j)\right]^{k}
$$

Definition 7: For a given texture $T$, the $N x 1$ vector $M^{a}(T)$, where the entries are the $\mu^{n}{ }_{z}$ (second order moment of $L^{n}(i j)$ ), for all the $n^{\text {th }}$ order clique chains, are called the second order moment vector.

$\underline{M}^{\mathrm{n}}(\mathrm{T})$ is used to represent texture $T$. At this point, any distance (Euclidean, Mahalanobis, Yule, Jakard etc.) can be used to measure the similarity between the textures by using the $\underline{M}^{n}(T)$ vectors.

For examples, the second order moment vector of the second order clique chains for texture $T$ is $\underline{M}^{2}(T)=\left\{\mu_{12}^{2}, \mu_{13}^{2}, \mu_{14, \ldots,}^{2} \mu_{78}^{2}\right\} . \underline{M}^{2}(T)$ gives only a rough idea about the texture appearance. However, as the order $n$ gets larger, the second order moment vector provides a more detailed information, with the price of increasing the dimension of $\underline{M}^{\mathrm{n}}(T)$. In the following, an algorithm is proposed to compute $\mu_{z}^{\mathrm{n}}$ or each $\mathrm{n}^{\text {th }}$ order clique chain. It is clear that $\mathrm{O}^{2}{ }_{15}, \mathrm{O}^{2}{ }_{26}, \mathrm{O}^{2}{ }_{37}, \mathrm{O}^{2}{ }_{48}$ clique chains are line like. For these types of clique chains, we replace the length of clique chain formulas to $L_{P Q}{ }^{2}(i j)=L_{P}(i j)+L_{Q}(i j)$.

\section{EXPERIMENTAL RESULTS}

In our simulation experiments, first, second, third and fourth order MRF models are used for modeling binary and gray level images. Textures of size $64 \times 64$ are 
generated according to various settings of MRF parameters. The algorithm is implemented under $\mathrm{C}$ programming language.

The second order moments for a given texture are scaled to 1 by dividing all the second order moments to the value of the maximum second order moments of the texture. This scaling provides invariancy in size and dimension of texels. As a next step, the distance of second order moments are used to calculate the distance between the textures $\mathrm{T} 1$ and $\mathbf{T} 2$ is, then, given as $\mathrm{d}(\mathrm{x}, \mathrm{y})=\mid \underline{\mathbf{M}}^{\mathrm{n}}$ (T1)- $\underline{\mathbf{M}}^{\mathrm{n}}(\mathrm{T} 2) \mid$

The above distance gives us a measure for the similarity of texture $\mathbf{T} 1$ and $\mathbf{T} 2$. Using this distance, it is possible to classify the textures. Experimental results show that $\mu_{z}^{n}$ very successful for identifying visually similar textures. Table 2 shows the most similar five textures according to the Euclidean distance of second order moments. Visual inspection of the textures 1-30 (Figure 3) and the quantitative analysis of table 2 indicate that the proposed distance is highly consistent with our human visual system in measuring the texture similarity.

\section{CONCLUSIONS}

In this paper, second order moments of $\mathrm{L}^{\mathrm{n}} \mathrm{z}(\mathrm{ij})$, are used to measure the similarity of binary and gray level Markov Random Fields textures. It is intuitively clear that the distribution of different clique types in the image defines texture. Therefore, by using the clique type statistics on a given texture, we can get an idea about the appearance of the texture.

Based on the above argument, we introduce the definitions for base clique, $\mathrm{n}^{\text {th }}$ order clique, second order moments with respect to origin of $\mathrm{L}^{\mathrm{n}}{ }_{\mathrm{z}}(\mathrm{ij})$. $\mu^{\mathrm{n}}{ }_{\mathrm{z}}$ is used to obtain quantitative values about the distribution of the different base cliques. Simulation examples demonstrate that $\mu^{n}{ }_{2}$ provide most of the information about the appearance of the texture of an image. The results are very consistent with the human visual system. In conclusion, using the second order statistics of the length of $n^{\text {th }}$ order clique chains, visually similar textures can be easily identified.

\section{REFERENCES}

1. Derin and $\mathrm{H}$. Elliott, "Modelling and segmentation of noisy and textured images using Gibbs random fields", IEEE Trans. Pat. Anal. Mach. Intel., vol. PAMI-9, pp. $39=55,1987$.

2. S.Lakshamanan and H. Derin, "Simultaneous parameter estimation and segmentation of Gibbs random fields simulated annealing", IEEE Trans. Pat. Anal. Mach. Intel., vol. 11., pp. 799 - 813, 1989.

3. S. Cohen and D. B. Cooper, "Simple parallel hierarchical and relaxation algorithms for segmenting noncausal Markovian random fields", IEEE Trans. Pat. Anal. Mach. Intel., vol. PAMI-9, pp. 195 - 219, 1987. 
4. Derin, "The use of Gibbs distributions in image processing", in Communications and Networks: A Survey of Recent Advances, I. Blake and V. Poor, Eds. New York: Springer - Verlag, 1985.

5. A. Carkacioglu and F.T. Yarman-Vural, "Set of Texture Similarity Measures", Electronic Imaging 97, Machine Vision Applications in Industrial Inspection, SPIE Proceedings Vol 3029, pp76-84 San Jose, 1997.

Table 1: The most similar three textures shown in Figure 3

\begin{tabular}{|c|c|c|c|c|c|c|}
\hline $\begin{array}{c}\text { Texture } \\
\text { No }\end{array}$ & $\begin{array}{c}\text { Texture } \\
\text { No }\end{array}$ & Distance & $\begin{array}{c}\text { Texture } \\
\text { No }\end{array}$ & Distance & $\begin{array}{c}\text { Texture } \\
\text { No }\end{array}$ & Distance \\
\hline 1 & 2 & 0.13 & 3 & 0.28 & 12 & 1.97 \\
\hline 2 & 1 & 0.13 & 3 & 0.20 & 5 & 1.95 \\
\hline 3 & 2 & 0.20 & 1 & 0.28 & 5 & 1.94 \\
\hline 4 & 6 & 0.28 & 5 & 0.29 & 11 & 1.96 \\
\hline 5 & 6 & 0.19 & 4 & 0.29 & 3 & 1.94 \\
\hline 6 & 5 & 0.19 & 4 & 0.28 & 12 & 1.95 \\
\hline 7 & 20 & 0.77 & 19 & 0.81 & 21 & 0.81 \\
\hline 8 & 19 & 0.30 & 20 & 0.35 & 21 & 0.35 \\
\hline 9 & 8 & 0.58 & 19 & 0.76 & 21 & 0.80 \\
\hline 10 & 12 & 0.77 & 11 & 0.80 & 8 & 1.94 \\
\hline 11 & 12 & 0.09 & 10 & 0.80 & 6 & 1.95 \\
\hline 12 & 11 & 0.09 & 10 & 0.77 & 6 & 1.95 \\
\hline 13 & 14 & 0.69 & 15 & 0.73 & 28 & 1.11 \\
\hline 14 & 13 & 0.69 & 15 & 1.00 & 28 & 1.31 \\
\hline 15 & 13 & 0.73 & 28 & 0.76 & 14 & 1.00 \\
\hline 16 & 17 & 0.87 & 18 & 0.90 & 25 & 2.46 \\
\hline 17 & 16 & 0.87 & 18 & 1.04 & 3 & 2.41 \\
\hline 18 & 16 & 0.90 & 17 & 1.04 & 5 & 2.42 \\
\hline 19 & 21 & 0.23 & 20 & 0.23 & 8 & 0.30 \\
\hline 20 & 21 & 0.18 & 19 & 0.23 & 8 & 0.35 \\
\hline 21 & 20 & 0.18 & 19 & 0.23 & 8 & 0.35 \\
\hline 22 & 24 & 2.21 & 23 & 2.61 & 26 & 3.97 \\
\hline 23 & 24 & 2.06 & 22 & 2.61 & 26 & 2.85 \\
\hline 24 & 26 & 1.96 & 23 & 2.06 & 22 & 2.21 \\
\hline 25 & 27 & 0.82 & 26 & 1.34 & 16 & 2.46 \\
\hline 26 & 25 & 1.34 & 27 & 1.51 & 24 & 1.96 \\
\hline 27 & 25 & 0.82 & 26 & 1.51 & 16 & 2.48 \\
\hline 28 & 15 & 0.76 & 30 & 0.91 & 13 & 1.11 \\
\hline 29 & 30 & 0.84 & 28 & 1.25 & 15 & 1.71 \\
\hline 30 & 29 & 0.84 & 28 & 0.91 & 15 & 1.28 \\
\hline
\end{tabular}



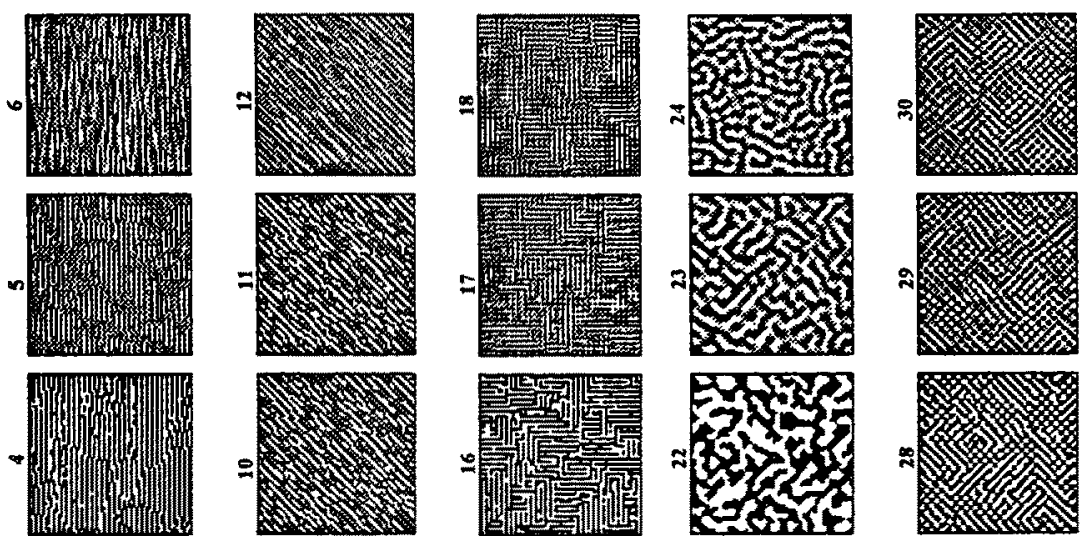

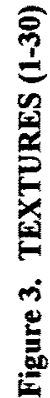
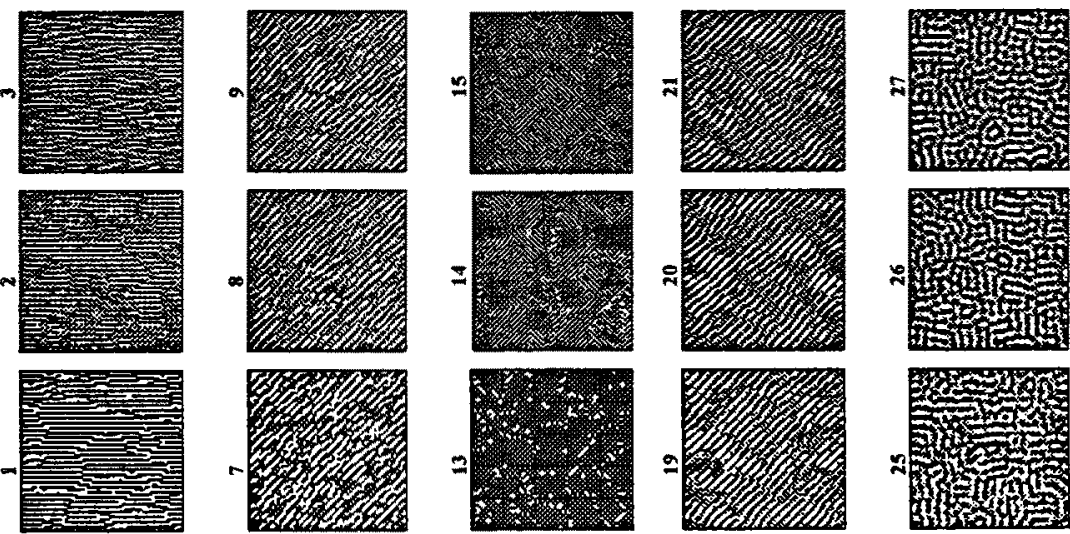